PAPER

\title{
Numerical investigation of effect of support conditions of poroelastic materials in impedance tube measurement
}

\author{
Naohisa Inoue* and Tetsuya Sakuma ${ }^{\dagger}$ \\ Graduate School of Frontier Sciences, The University of Tokyo, \\ Environ. Bldg., 5-1-5 Kashiwanoha, Kashiwa, 277-8563 Japan
}

(Received 1 June 2016, Accepted for publication 20 February 2017)

\begin{abstract}
It is well known that the support conditions of a specimen can significantly affect the results of the impedance tube measurement. In theory, the specimen should be mounted under the ideal slip condition without any circumferential air gaps. However, this cannot be fully realized due to the inaccuracy of material cutting, thus causing a constraint or gapped condition. Furthermore, the specimen is normally mounted lightly in contact with the bottom of the tube, or with a back air layer. In this paper, the effect of realistic support conditions for poroelastic materials is investigated by numerical simulation with the finite element method. Under each condition, the behaviors of calculated values of normal-incidence absorption coefficient and transmission loss are discussed by comparison with theoretical values for the infinite-area material. It is shown that several mechanical and acoustical parameters of poroelastic materials can be estimated from impedance tube measurement under the circumferential constraint and gapped conditions.
\end{abstract}

Keywords: Impedance tube, Poroelastic material, Support condition, Biot's theory, Finite element method

PACS number: 43.55.Ev [doi:10.1250/ast.38.213]

\section{INTRODUCTION}

Impedance tube measurement (ITM) is widely used to measure various acoustical properties of porous materials such as the surface impedance, absorption coefficient, transmission loss, characteristic bulk properties, and so on. Some measurement methods for the above properties have been standardized by ISO 10534-2 [1] based on the rigidframe assumption for porous material. However, in practical application, it is well known that the circumferential condition of a specimen can significantly affect the results of ITM. The specimen should be mounted under the ideal slip condition and without any air gaps to the lateral faces of the tube to measure the acoustic characteristics that are equivalent to those for the infinite-area material. However, this cannot be fully realized due to the inaccuracy of material cutting for loose fitting. Therefore, a constraint, gapped, or their mixed condition is likely to arise, which often leads to discrepancies between measured and theoretical values.

Several papers have been dedicated to the theoretical and numerical solution of circumferential constraint and

\footnotetext{
*e-mail: inoue@env-acoust.k.u-tokyo.ac.jp

†e-mail: sakuma@k.u-tokyo.ac.jp
}

gapped problems in ITM. For the circumferential constraint condition, Kang and Bolton investigated its effects on the absorption coefficient and surface impedance of poroelastic foam with an open or membrane-sealed surface by the two-dimensional (2-D) finite element method (FEM) [2]. It was concluded that the primary effect is to increase the apparent rigidity of poroelastic materials, and which becomes pronounced for a narrower tube. Furthermore, Vigran et al. and Song et al. also referred to the apparent stiffening effect and the shear vibration mode excited in the material $[3,4]$. Moreover, Pilon et al. developed a criterion to estimate the deviations from theoretical values under the circumferential constraint condition through numerical calculation conducted for a large number of foam samples [5]. However, this criterion is not always available because it requires physical properties of a specimen, which are rarely known before ITM.

For the circumferential gapped condition, Cummings investigated its effects experimentally, and also analytically on the assumption that the sound pressure is distributed uniformly over the cross section in the tube [6]. This assumption is valid for materials with low flow resistivity. It was concluded that circumferential air gaps mainly affect the real part of the surface impedance in the low- and mid-frequency range. On the other hand, Pilon 
Table 1 Combinations of the circumferential and bottom conditions simulated by TMM and FEM.

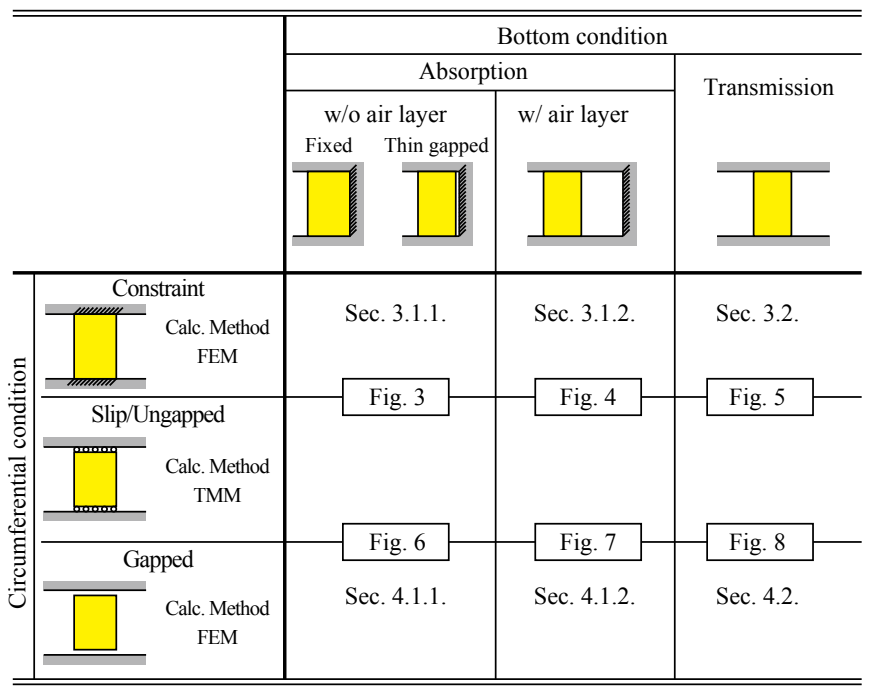

et al. referred to the effects of gaps for materials with high flow resistivity in the context of the double porosity theory (DPT), and again developed a criterion and charts to estimate the deviation from theoretical values $[7,8]$.

In the above references, the effect of the back condition of a specimen was not sufficiently discussed. Pilon's criteria were developed for the case where a specimen is fixed on the bottom face of the tube. However, in actual measurements, specimens are rarely fixed and are lightly in contact with the bottom. Moreover, in the case with an air layer behind a specimen, the effects of the circumferential constraint and gapped conditions are not yet clarified. In this paper, numerical simulation with the three dimensional (3-D) FEM is executed to investigate the effects of circumferential conditions in practical measurement arrangements of a material and back air layer. The possibility of estimating several acoustical and mechanical parameters of poroelastic materials is also presented through case studies.

\section{NUMERICAL MODELING}

Table 1 shows the combinations of circumferential and bottom conditions investigated in the following sections. For the specimen mounted in the circumferential slip and ungapped condition, sound and vibration fields in the tube are theoretically equivalent to the normal-incidence state for the infinite-area material. Accordingly, acoustical indicators under the condition are calculated by the transfer matrix method (TMM) [9]. On the other hand, the FEM is employed for the calculation of indicators under the circumferential constraint and gapped conditions. This section presents formulations for the FEM and calculation conditions. The domain notations are given in Fig. 1. In the following, the values with $\delta$ are the admissible variations for unknowns and $j$ is the imaginary unit.

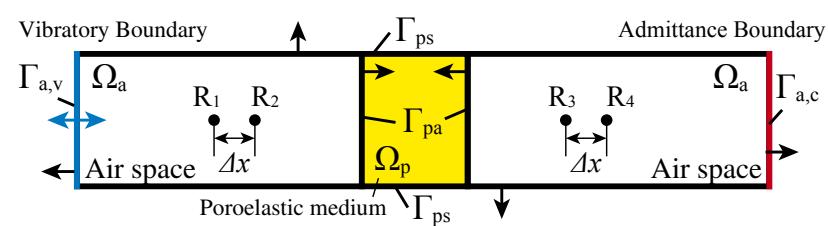

Fig. 1 Impedance tube model for calculating transmission loss with four receivers.

\subsection{Weak-Form Representation}

In an air space, the integral equation in the weak form is given by

$$
\begin{gathered}
\int_{\Omega_{\mathrm{a}}}\left(\nabla \delta p^{\mathrm{a}} \cdot \nabla p^{\mathrm{a}}\right) d V-k_{0}^{2} \int_{\Omega_{\mathrm{a}}} \delta p^{\mathrm{a}} p^{\mathrm{a}} d V-\int_{\Gamma_{\mathrm{pa}}} \delta p^{\mathrm{a}} \frac{\partial p^{\mathrm{a}}}{\partial n} d S \\
-j \omega \rho_{0} \int_{\Gamma_{\mathrm{a}, \mathrm{v}}} \delta p^{\mathrm{a}} v_{\mathrm{f}} d S+j k_{0} \beta_{\mathrm{n}} \int_{\Gamma_{\mathrm{a}, \mathrm{c}}} \delta p^{\mathrm{a}} p^{\mathrm{a}} d S=0
\end{gathered}
$$

where $\rho_{0}, k_{0}, \omega$ and $p^{\mathrm{a}}$ are the air density, wave number, angular frequency, and sound pressure, respectively. $v_{\mathrm{f}}$ is the vibration velocity of the source surface $\Gamma_{\mathrm{a}, \mathrm{v}} \cdot v_{\mathrm{f}}$ is set as $1 \mathrm{~m} / \mathrm{s}$ in all calculations. $\beta_{\mathrm{n}}$ is the normalized admittance of the bottom surface of the transmission-side tube $\Gamma_{\mathrm{a}, \mathrm{c}} . \beta_{\mathrm{n}}$ is set as 0 for absorption problems and 1 for transmission problems.

Biot's theory is a poroelastic model that describes the interactive propagation of elastic waves in solid and fluid phases of porous media. The weak-form integral equations of the mixed displacement-pressure representation of the Biot's theory are given as [10]

$$
\begin{aligned}
& \int_{\Omega_{\mathrm{p}}} \delta \underline{\underline{\underline{\varepsilon}}}: \underline{\underline{\sigma}}^{\mathrm{s}}\left(\boldsymbol{u}^{\mathrm{s}}\right) d V-\tilde{\rho}_{s} \omega^{2} \int_{\Omega_{\mathrm{p}}} \delta \boldsymbol{u}^{\mathrm{s}} \cdot \boldsymbol{u}^{\mathrm{s}} d V \\
& \quad-\tilde{\gamma} \int_{\Omega_{\mathrm{p}}} \delta \boldsymbol{u}^{\mathrm{s}} \cdot \nabla p d V \\
& \quad-\int_{\Gamma_{\mathrm{pa}}, \Gamma_{\mathrm{ps}}}\left[\delta \boldsymbol{u}^{\mathrm{s}} \cdot\left(\underline{\underline{\sigma^{\mathrm{t}}}} \cdot \boldsymbol{n}\right)+\left(\delta \boldsymbol{u}^{\mathrm{s}} \cdot \boldsymbol{n}\right) p\right] d S=0, \\
& \frac{\phi^{2}}{\tilde{\rho}_{22} \omega^{2}} \int_{\Omega_{\mathrm{p}}} \nabla \delta p \cdot \nabla p d V-\frac{\phi^{2}}{\tilde{R}} \int_{\Omega_{\mathrm{p}}} \delta p \cdot p d V \\
& \quad-\tilde{\gamma} \int_{\Omega_{\mathrm{p}}} \nabla \delta p \cdot \boldsymbol{u}^{\mathrm{s}} d V \\
& \quad-\int_{\Gamma_{\mathrm{pa}}, \Gamma_{\mathrm{ps}}}\left[\delta p \cdot \phi\left(u_{\mathrm{n}}^{\mathrm{f}}-u_{\mathrm{n}}^{\mathrm{s}}\right)+\delta p \cdot u_{\mathrm{n}}^{\mathrm{s}}\right] d S=0,
\end{aligned}
$$

where $\boldsymbol{u}^{\mathrm{s}}$ and $p$ are the solid-phase displacement and fluidphase sound pressure, and $u_{\mathrm{n}}^{\mathrm{s}}$ and $u_{\mathrm{n}}^{\mathrm{f}}$ are the outward normal displacements of the solid and fluid phases on the boundary respectively. $\boldsymbol{n}$ denotes the unit normal vector on the interface. In this paper, materials are assumed to be incompressible in the scale of the fiber frame. Accordingly, the following values are introduced for the physical parameters. In the fluid phase, 
- $\tilde{\rho}_{\mathrm{f}}, \quad \tilde{K}_{\mathrm{f}}$ : effective density and bulk modulus of equivalent fluid,

- $\tilde{\rho}_{22}, \tilde{R}$ : Biot's inertial and elastic coefficients for fluid phase defined by $\tilde{\rho}_{22}=\phi \tilde{\rho}_{\mathrm{f}}$ and $\tilde{R}=\phi \tilde{K}_{\mathrm{f}}$.

In the solid phase,

- $\rho_{\mathrm{b}}, K_{\mathrm{b}}$ : bulk density and bulk modulus of poroelastic material in vacuo,

- $\tilde{\rho}_{\mathrm{s}}$ : effective solid phase density defined by $\tilde{\rho}_{\mathrm{s}}=$ $\rho_{\mathrm{b}}+\phi \rho_{0}\left(1-\rho_{0} / \tilde{\rho}_{\mathrm{f}}\right)$

- $\lambda, \mu$ : Lamé's elastic coefficients,

- $\underline{\underline{\varepsilon}}^{\mathrm{s}}$ : strain tensor of the solid phase of poroelastic material given by $\varepsilon_{i j}^{\mathrm{s}}=1 / 2\left(\partial u_{i}^{\mathrm{s}} / \partial x_{j}+\partial u_{j}^{\mathrm{s}} / \partial x_{i}\right)$,

- $\underline{\underline{\sigma}}^{\mathrm{s}}$ : stress tensor of the poroelastic material in vacuo; it is related to the strain tensor by Hooke's law together with the unit tensor $\underline{\underline{\mathrm{I}}}$ by $\underline{\underline{\sigma}}^{\mathrm{s}}=\lambda \operatorname{div} \boldsymbol{u}^{\mathrm{S}} \underline{\underline{\mathrm{I}}}+2 \mu \underline{\underline{\underline{\varepsilon}}} \mathrm{s}$,

- $\underline{\underline{\sigma}}^{\mathrm{t}}$ : total stress tensor in the poroelastic material defined by $\underline{\underline{\sigma}}^{\mathrm{t}}=\underline{\underline{\sigma}}^{\mathrm{s}}-p \underline{\underline{\mathrm{I}}}$,

In both phases

- $\varphi$ : porosity of the poroelastic material,

- $\tilde{\gamma}$ : volumetric coupling coefficient defined by $\tilde{\gamma}=$ $\phi\left(\rho_{0} / \tilde{\rho}_{\mathrm{f}}\right)-1$.

The values with a tilde in the above are complex and frequency-dependent. $\tilde{\rho}_{\mathrm{f}}$ and $\tilde{K}_{\mathrm{f}}$ are evaluated with the Johnson-Champoux-Allard model [11] in this paper.

In the case of a rigid-frame material, i.e., $\boldsymbol{u}^{\mathrm{s}}=0$, Eq. (2) and the third term of Eq. (3) become zero and the finite element implementation becomes identical to Craggs' method [12].

\subsection{Continuity Conditions between Air Space and Poroelastic Material}

The continuity conditions on the interface $\Gamma_{\mathrm{pa}}$ between an acoustic field in an air space and a vibro-acoustic field in a poroelastic material are given as [13]

$$
\left\{\begin{array}{l}
\frac{\sigma^{\mathrm{t}}}{\bar{p}} \cdot \boldsymbol{n}=-p^{\mathrm{a}} \cdot \boldsymbol{n} \\
(1-\phi) u_{\mathrm{n}}^{\mathrm{s}}+\phi u_{\mathrm{n}}^{\mathrm{f}}=\frac{1}{\rho_{0} \omega^{2}} \frac{\partial p^{\mathrm{a}}}{\partial n} .
\end{array}\right.
$$

The boundary integral terms for the interface vanish under these conditions. In the finite element implementation, only the second equation is imposed explicitly by using common unknowns for nodal pressures on the interface.

\subsection{Boundary Conditions for Poroelastic Material}

The fixed support conditions for a lateral boundary $\Gamma_{\mathrm{ps}}$ are represented as

$$
\left\{\begin{array}{l}
\boldsymbol{u}^{\mathrm{s}}=0 \\
u_{\mathrm{n}}^{\mathrm{s}}-u_{\mathrm{n}}^{\mathrm{f}}=0
\end{array}\right.
$$

The boundary integral terms in Eqs. (2) and (3) vanish under these conditions. Thus, the fixed support condition is fulfilled by eliminating the degrees of freedom for solid-

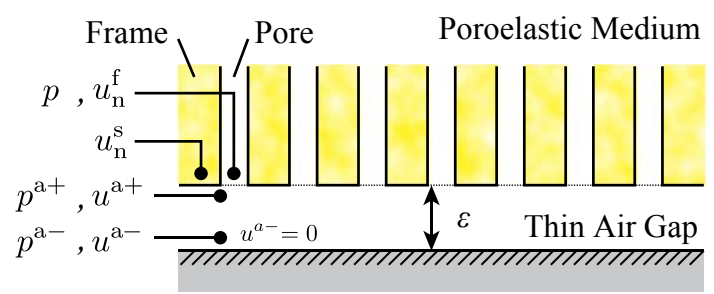

Fig. 2 Poroelastic material placed close to the rigid wall with thin air gap.

phase displacements on the boundary in the finite element implementation.

Subsequently, a simple model for a bottom thin gapped condition is derived in this paper as depicted in Fig. 2. The Continuity conditions on the interface between the air gap and poroelastic medium are given as Eq. (4). If the gap becomes vanishingly thin, the sound pressures and their normal derivatives on the forward and backward faces will coincide. Furthermore, assuming rigid backing, thin-gap condition leads to

$$
\left\{\begin{array}{l}
\underline{\underline{\sigma}}^{\mathrm{t}} \cdot \boldsymbol{n}=-p \cdot \boldsymbol{n} \\
(1-\phi) u_{\mathrm{n}}^{\mathrm{s}}+\phi u_{\mathrm{n}}^{\mathrm{f}}=0 .
\end{array}\right.
$$

Under these conditions, the boundary integral terms in Eqs. (2) and (3) become zero and no additional procedure is required for the finite element implementation. However, it is emphasized that this condition is not applicable to lateral gaps where the sound pressure and particle velocity can rapidly vary from an interface between a material and the air gap to the opposite tube face. Thus, in the numerical analyses conducted in this paper, a bottom thin gap was modeled under the condition described above, whereas circumferential gaps were modeled by creating acoustic elements.

\subsection{Calculation Conditions}

In our preliminary numerical study [14], it was confirmed that the differences in absorption and transmission characteristics are small between the tubes with hexadecagon and square cross sections of an equal area. In this paper, a square tube with a side length $L$ of $50 \mathrm{~mm}$ was used, where the equivalent diameter of a circular tube is given by $D_{\text {eq }}=1.13 \times L$. In conventional ITM, the upperlimit frequency of this tube is about $3,400 \mathrm{~Hz}$. However, in the numerical calculation, two plane symmetries are assumed for the vibration surface and the material, thus doubling the upper-limit frequency. The calculation domains are discretized by 27-node second-order hexahedral elements, whose maximal lengths are 20 and $10 \mathrm{~mm}$ for the air space and poroelastic material domains, respectively. Calculations are conducted at the 1/24-octave center frequencies from 63 to $5,040 \mathrm{~Hz}$. 
Table 2 Physical properties of the tested glass wool.

\begin{tabular}{cccc}
\hline Property name & Symbol & Value & Dimension \\
\hline Shear modulus & $N$ & 100,000 & {$\left[\mathrm{~N} / \mathrm{m}^{2}\right]$} \\
Poisson's ratio & $v$ & 0 & {$[\mathrm{]}$} \\
Loss factor & $\eta$ & 0.25 & {$[\mathrm{]}$} \\
Bulk density & $\rho_{\mathrm{b}}$ & 96 & {$\left[\mathrm{~kg} / \mathrm{m}^{3}\right]$} \\
Flow resistivity & $\sigma$ & 55,000 & {$\left[\mathrm{Ns} / \mathrm{m}^{4}\right]$} \\
Porosity & $\phi$ & 0.96 & {[]} \\
Tortuosity & $\alpha_{\infty}$ & 1.07 & {[]} \\
Viscous characteristic length & $\Lambda$ & 50 & {$[\mu \mathrm{m}]$} \\
Thermal characteristic length & $\Lambda^{\prime}$ & 100 & {$[\mu \mathrm{m}]$} \\
\hline
\end{tabular}

After solving the system matrix equation, the normalincidence absorption coefficient $\alpha_{\mathrm{n}}$ and the transmission loss $R_{\mathrm{n}}$ are obtained by the two- and four-microphone methods as follows:

$$
\begin{aligned}
\alpha_{\mathrm{n}} & =1-\left|\frac{p_{2}-p_{1} \exp \left(j k_{0} \Delta x\right)}{p_{1} \exp \left(-j k_{0} \Delta x\right)-p_{2}}\right|^{2}, \\
R_{\mathrm{n}} & =-20 \log _{10}\left|\frac{p_{3} \exp \left(j k_{0} \Delta x\right)-p_{4}}{p_{1} \exp \left(j k_{0} \Delta x\right)-p_{2}}\right|,
\end{aligned}
$$

where $p_{1}$ to $p_{4}$ are the complex sound pressures at the receivers $R_{1}$ to $R_{4}$, respectively (see Fig. 1). $\Delta x$ is the interval between each pair of receivers and is set as $30 \mathrm{~mm}$. Thus, the singular frequency at which progressive and regressive waves cannot be separated is about $5,700 \mathrm{~Hz} . R_{2}$ and $R_{3}$ are located more than $0.1 \mathrm{~m}$ away from the material surfaces $\Gamma_{\mathrm{pa}}$ to reduce the effect of evanescent waves.

According to Biot's theory and the DPT, the effects of the circumferential constraint and gapped conditions become pronounced for materials with high flow resistivity. Therefore, case studies were conducted for a glass wool and a urethane foam with relatively high flow resistivity. As similar tendencies were observed for the effect of the support conditions [15], this paper only gives the results for the glass wool and discusses the tendencies. The physical properties of the glass wool are listed in Table 2.

\section{BEHAVIOR UNDER THE CIRCUMFERENTIAL CONSTRAINT CONDITION}

\subsection{Absorption Coefficient}

Calculations in this section are conducted for all combinations of material thicknesses $(12.5,25$, and 50 $\mathrm{mm})$ and air layer thicknesses $(0,12.5,25$, and $50 \mathrm{~mm})$. In the following, the elastic frame characteristics under the circumferential slip condition are first interpreted. Then, the effects of the circumferential constraint are discussed.

\subsubsection{Cases without air layer}

Figure 3 shows results for materials mounted without the air layer. Features for the circumferential slip condition (upper figures) are as follows. Under the bottom fixed condition, dips appear around the frequencies, $f_{1}$, at which the quarter-wavelength longitudinal resonances of the frame occur in the thickness direction. Under the bottom thin-gapped condition, a peak caused by the frame resonance is seen around $500 \mathrm{~Hz}$ for the 50 -mm-thickness material. The frequency corresponds to that of the halfwavelength longitudinal mode resonance.

Subsequently, results for the circumferential constraint condition (lower figures) are discussed below. Under the bottom fixed condition, dip frequencies are shifted slightly higher from $f_{1}$ for 12.5 and $25 \mathrm{~mm}$ thicknesses. For 50-mmthick material, the resonance appears to occur around $f_{\mathrm{s}}$, the frequency of $(1,1)$ shear mode in the cross section.

$f_{\mathrm{s}}$ is calculated by $f_{\mathrm{s}}=c_{\mathrm{s}} / \sqrt{2} L$, where $c_{\mathrm{s}}$ is the phase velocity of the transverse wave in the frame. Under the bottom thin-gapped condition, almost the same characteristics are observed as those for bottom fixed condition. However, the effects of the frame resonances appear at slightly lower frequencies for the 25- and 50-mm-thick materials.

\subsubsection{Cases with air layer}

Figure 4 shows the results for materials mounted with air layers. In the upper figures (the circumferential slip condition), it is clearly observed for the 50-mm-thick material that the absorption coefficients increase from those for the rigid-frame model at around $160 \mathrm{~Hz}$ due to the mass-spring resonance system composed of the material and the air layer.

In the lower figures, for the 12.5-mm-thickness material, dips occur around $f_{\mathrm{b}}$, the bending resonance frequency for a thick circular plate with diameter $D_{\text {eq }}$. On the other hand, the effects of the frame resonances appear around $f_{\mathrm{s}}$ for the 25- and 50-mm-thick materials. These tendencies imply that as the material becomes thicker, its deformation becomes far from bending. Regardless of the resonance mechanisms, below the first frame resonance frequency, the solid phase is considered to be under stiffness control, and the one-body vibration of the poroelastic material is restrained. Consequently, the absorption coefficients approach those for the rigid-frame model, and the discrepancy between the circumferential slip and constraint conditions becomes more pronounced around the mass-spring resonance frequencies. Furthermore, for all cases in Figs. 3 and 4, there are almost negligible differences between the characteristics for elastic- and rigid-frame models above $1,000 \mathrm{~Hz}$.

Moreover, according to the above observations, the shear modulus may be estimated as $N=2 L^{2} f_{\mathrm{s}}^{2} \rho_{\mathrm{b}}$ from the resonance frequency by using a thick specimen with a circumferential constraint. The requirements and validity of this estimation procedure must be studied further in future work. However, there is a tendency that the frame resonance occurs at $f_{\mathrm{s}}$ if the fundamental resonance 


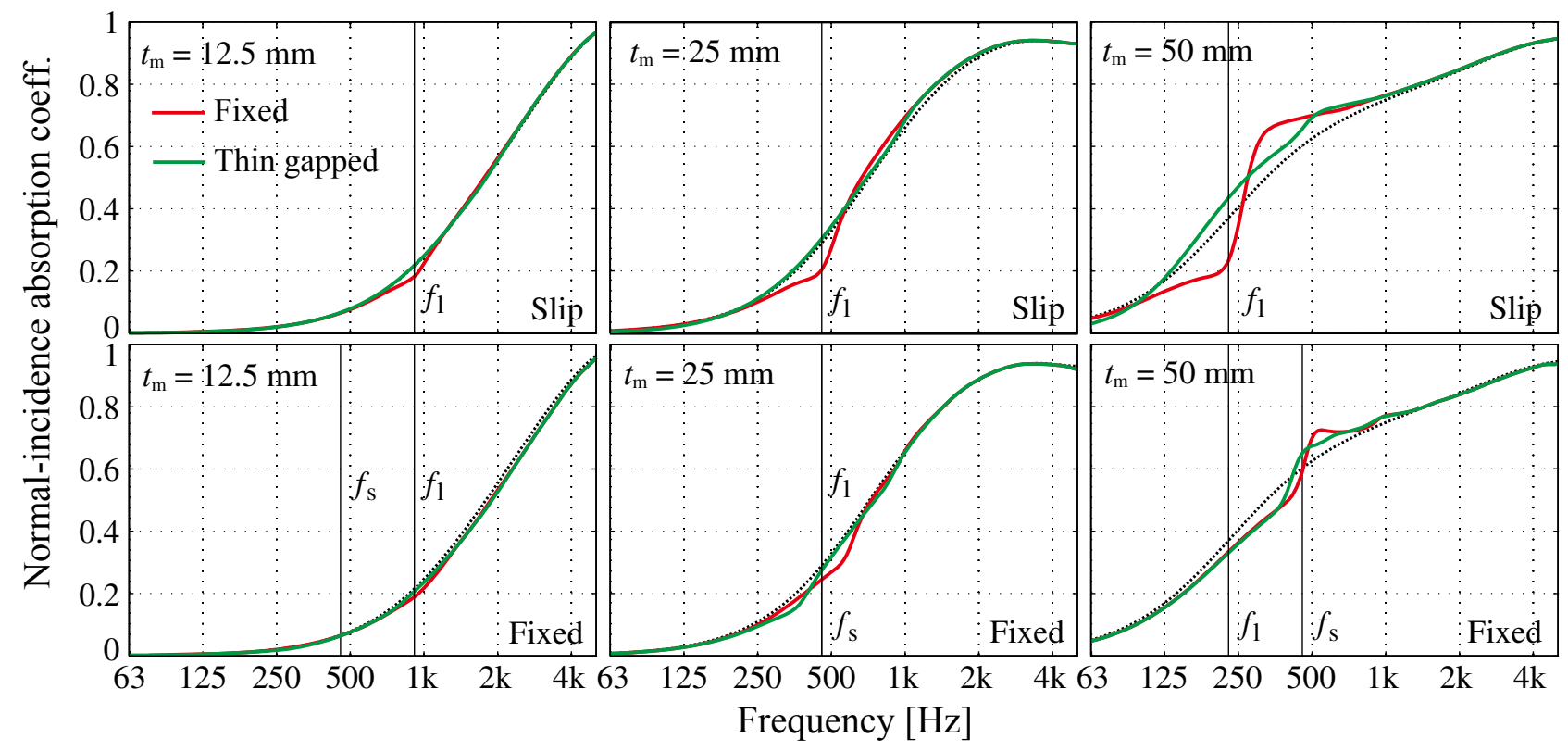

Fig. 3 Normal-incidence absorption coefficients calculated for the materials mounted without the air layer. Dotted lines are the characteristics for the rigid-frame model. The circumferential conditions are slip in the upper figures and constraint in the lower figures.

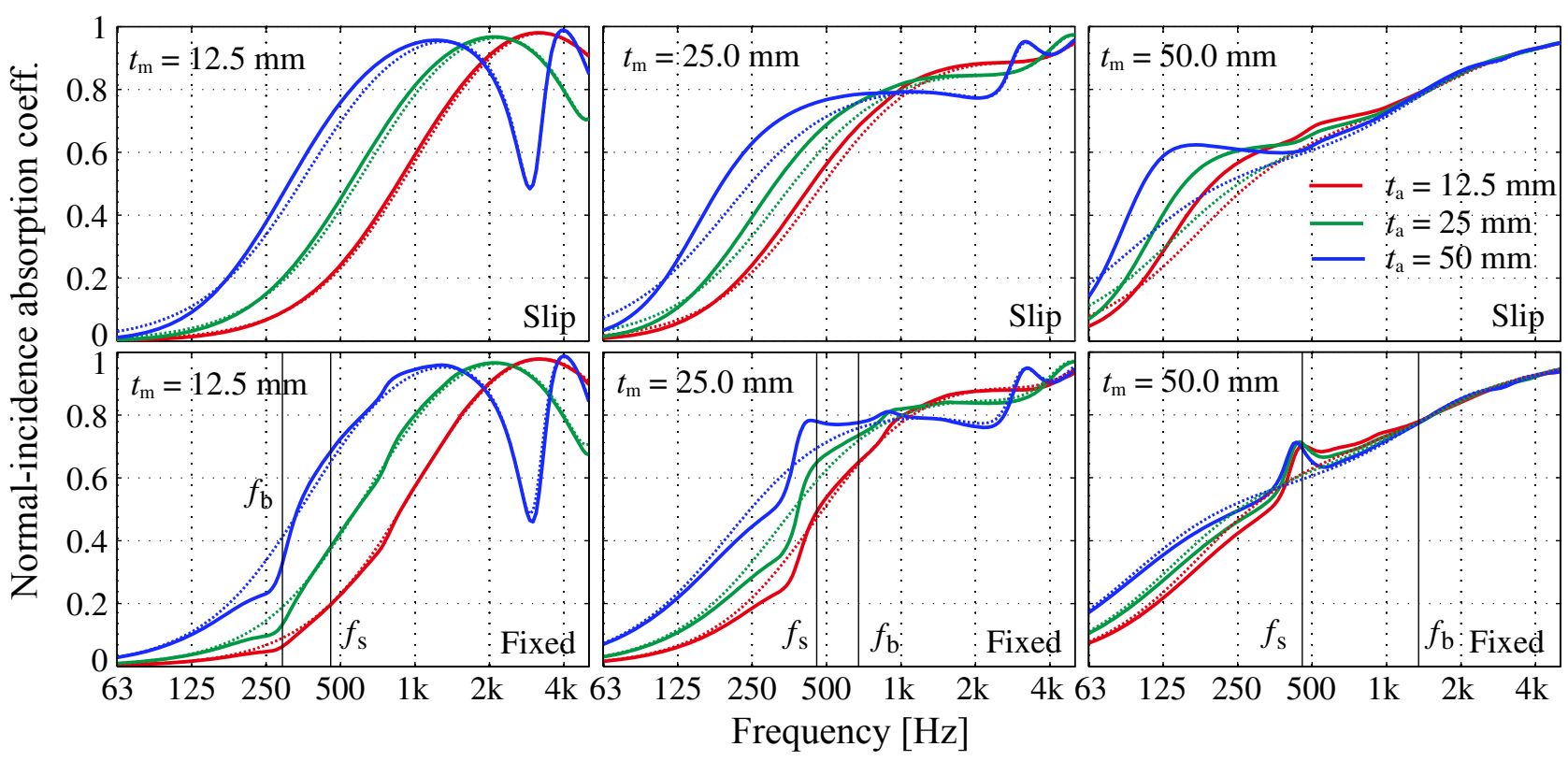

Fig. 4 Normal-incidence absorption coefficients calculated for the materials mounted with the air layer. Dotted lines are the characteristics for the rigid-frame model. The circumferential conditions are slip in the upper figures and constraint in the lower figures.

frequency of the thick bending plate $f_{\mathrm{b}}$ becomes much greater than that of the shear mode $f_{\mathrm{s}}$.

\subsection{Transmission Loss}

Figure 5 shows transmission losses calculated for the materials with $12.5,25$, and $50 \mathrm{~mm}$ thicknesses. The corresponding mass-law lines are also shown as thin black lines. Firstly, around $63 \mathrm{~Hz}$, all specimens have flat characteristics for the transmission loss. From the phenomenological fact that effective density approaches $\sigma / j \omega$ at low frequencies, the limit value of the transmission loss of a porous rigid material at a low frequency is theoretically given as

$$
R_{\mathrm{n}}=20 \log _{10}\left|1+\frac{\sigma t_{\mathrm{m}}}{2 \rho_{0} c_{0}}\right| \quad(\omega \rightarrow 0) .
$$




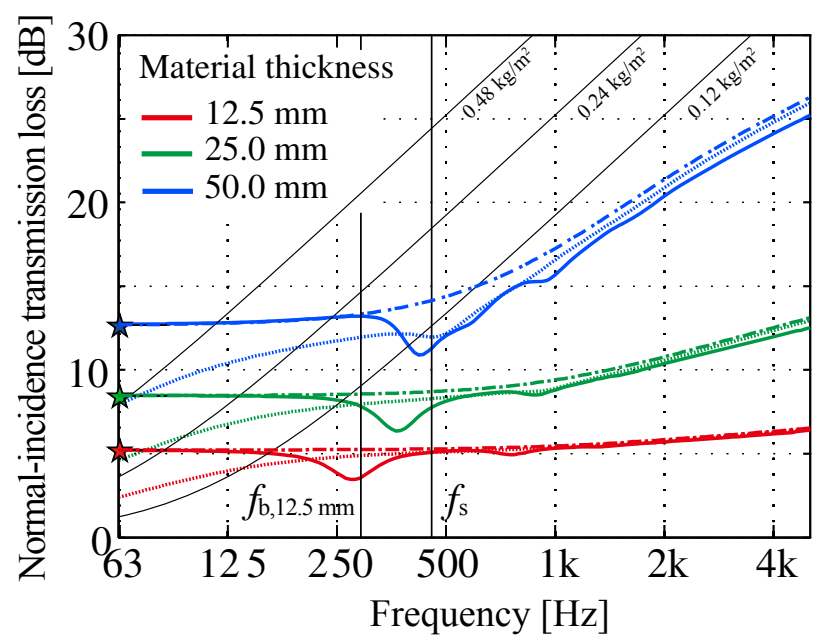

Fig. 5 Normal-incidence transmission losses calculated for materials with different thicknesses under the circumferential constraint condition. Solid and dotted lines are characteristics for the elastic frame with the circumferential constraint and slip conditions, respectively. Chain lines are characteristics for the rigid frame.

The limit for each material thickness is shown at $63 \mathrm{~Hz}$ with the star mark. In the high-frequency range, the transmission losses are considered to be due to attenuation in a material.

Secondly, in the results for the elastic frame material under the circumferential slip condition, the one-body vibration of the specimen is taken into account. Accordingly, the transmission losses decrease from the flow resistance controlled limits and approach to the mass law.

Finally, in the results for the elastic frame material under the circumferential constraint condition, here again, dips of the transmission loss appear around $f_{\mathrm{b}}$ for $12.5 \mathrm{~mm}$ thickness and $f_{\mathrm{s}}$ for $50 \mathrm{~mm}$ thickness. As mentioned in the previous section, below the first frame resonance frequencies, the solid phase of the specimen is under stiffness control, and transmission losses approach those for the rigid-frame model. Accordingly, Eq. (9) can be used to estimate the flow resistivity when the poroelastic material is supported rigidly on the side wall of the tube.

\section{BEHAVIOR UNDER THE CIRCUMFERENTIAL GAPPED CONDITION}

\subsection{Absorption Coefficient}

Results for the circumferential ungapped condition are identical to those for the circumferential slip condition in the previous section. Therefore, only the effects of the circumferential gap on the normal incidence absorption coefficient are discussed here. In the following, the macroporosity is defined as the ratio of the cross-section area of the circumferential gap to that of the tube. In all calculations, the macroporosity is set to 1,2 , and $4 \%$.

\subsubsection{Cases without air layer}

Figure 6 shows normal-incidence absorption coefficients calculated for materials with different thicknesses mounted close to the hard backing. Three material thicknesses, 12.5, 25, and $50 \mathrm{~mm}$, are considered.

Figure 6(a) shows the results for rigid-frame materials. Following DPT and Pilon's work [7,8], the frequencies, $f_{\mathrm{dp}}$, at which the quarter-wavelengths in the material become equal to the material thickness are numerically calculated for cases (a-2) and (a-3). For case (a-1), valid $f_{\text {dp }}$ cannot be obtained because the quarter-wavelengths appear in the region where DPT is no longer applicable. The absorption coefficients appear to decrease below and increase around $f_{\mathrm{dp}}$. These tendencies become more pronounced as the macroporosity increases. Furthermore, the discrepancy between gapped and ungapped conditions generally becomes smaller in the high-frequency range.

When the frame vibration is taken into account, materials with a thin bottom gap show almost the same characteristics as those for the rigid-frame model (compare Figs. 6(b) and 6(a)). However, the frequency at which the decrease in the absorption coefficient changes to an increase is shifted slightly higher. Furthermore, for $50 \mathrm{~mm}$ specimen, the absorption coefficient around the peak slightly increases.

On the other hand, in the case of materials with a constrained bottom (Fig. 6(c)), dips caused by the longitudinal resonance are large. The locations of the dips are hardly affected by the degree of macroporosity. On the basis of this tendency, the longitudinal resonance frequency may give an estimation of Young's modulus as $E=16 t_{\mathrm{m}}^{2} f_{\mathrm{l}}^{2} \rho_{\mathrm{b}}$. Note that Poisson's ratio may also be obtained by comparing $N$ with $E$, estimated from circumferential constraint and gapped conditions, respectively.

4.1.2. Cases with air layer

Figure 7 shows normal-incidence absorption coefficients calculated for the material with air layers having different thicknesses and circumferential gaps. The material thickness is $25 \mathrm{~mm}$. The air layer thicknesses are set as $12.5,25$, and $50 \mathrm{~mm}$.

In both Figs. 7(a) and 7(b), the effects of circumferential gaps are similar to those for the hard-backing cases. However, as the back air layer becomes thicker, the discrepancies between gapped and ungapped characteristics become greater even if the gap size is small. Comparing Fig. 7(a-2) with Fig. 6(a-3), where the distance between the front surface of the specimen and the hard backing is $50 \mathrm{~mm}$, similar characteristics are observed. This implies that the energy absorption dominantly occurs in the region where the particle velocity is large in the double-porosity medium as well as in the single-porosity one. Therefore, the degree of discrepancy between the values under gapped and ungapped conditions strongly 
Gap Size $d$ (Macroporosity)

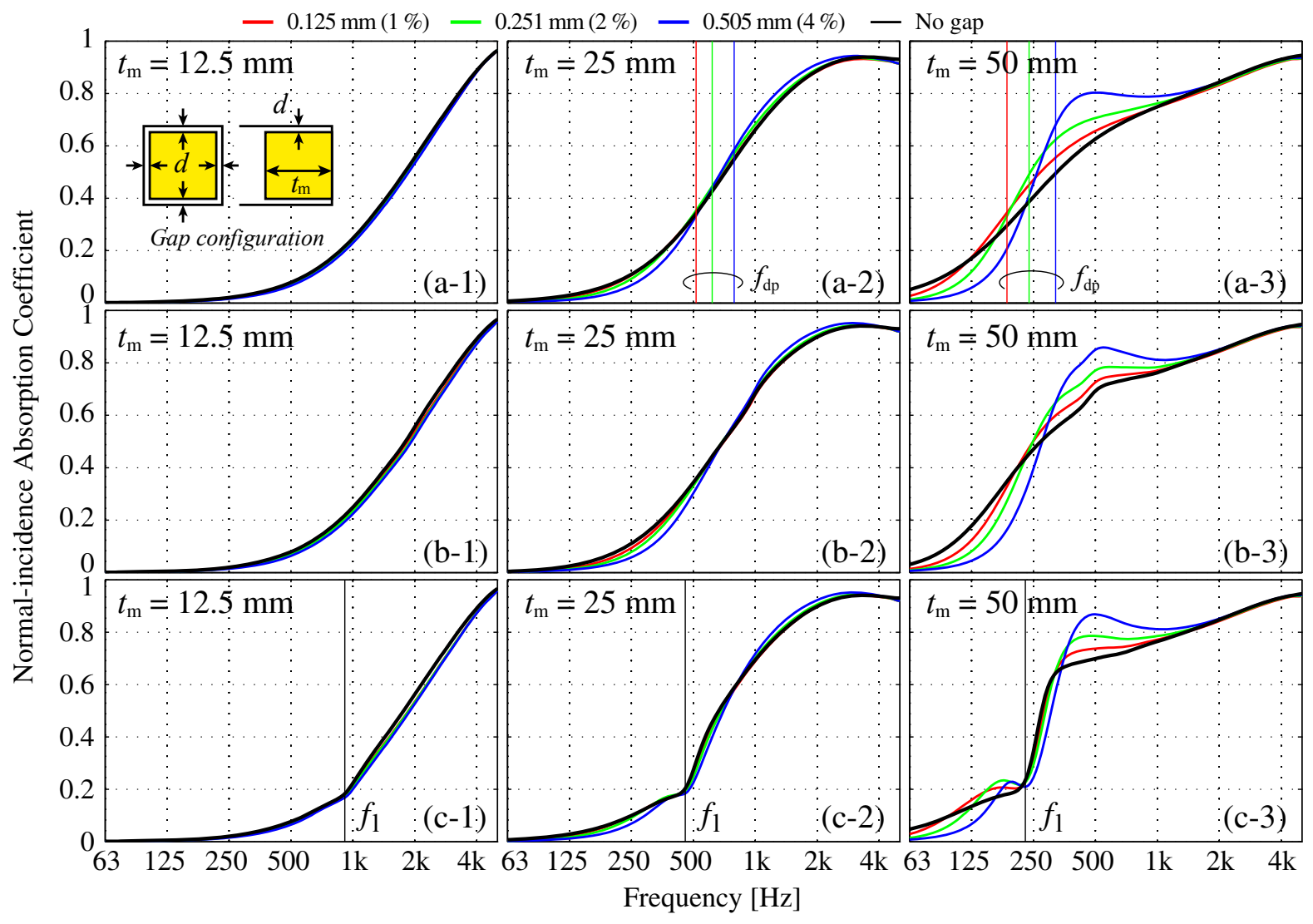

Fig. 6 Normal-incidence absorption coefficients calculated for the material mounted under the circumferential gapped condition without an air layer. The material thicknesses are (left) $12.5 \mathrm{~mm}$, (center) $25 \mathrm{~mm}$, and (right) $50 \mathrm{~mm}$. The materials are (a) a rigid frame, (b) an elastic frame with a thin bottom gap, and (c) an elastic frame with a bottom fixed condition.

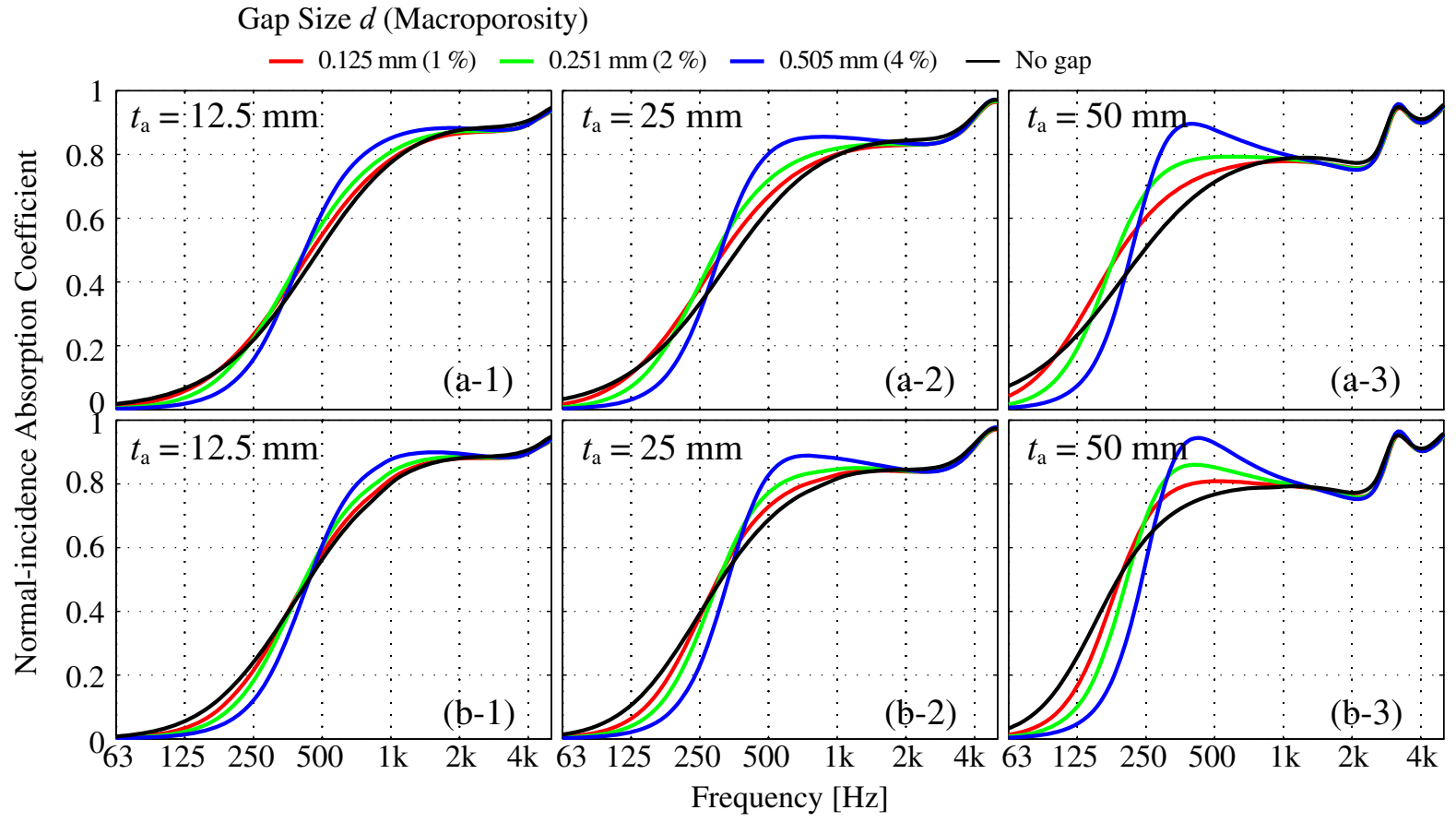

Fig. 7 Normal-incidence absorption coefficients calculated for the 25-mm-thickn material under the circumferential gapped condition. The material is backed by an air layer with different thicknesses: (left) $12.5 \mathrm{~mm}$, (center) $25 \mathrm{~mm}$, and (right) $50 \mathrm{~mm}$. The frame of the material is assumed to be (a) rigid and (b) elastic. 


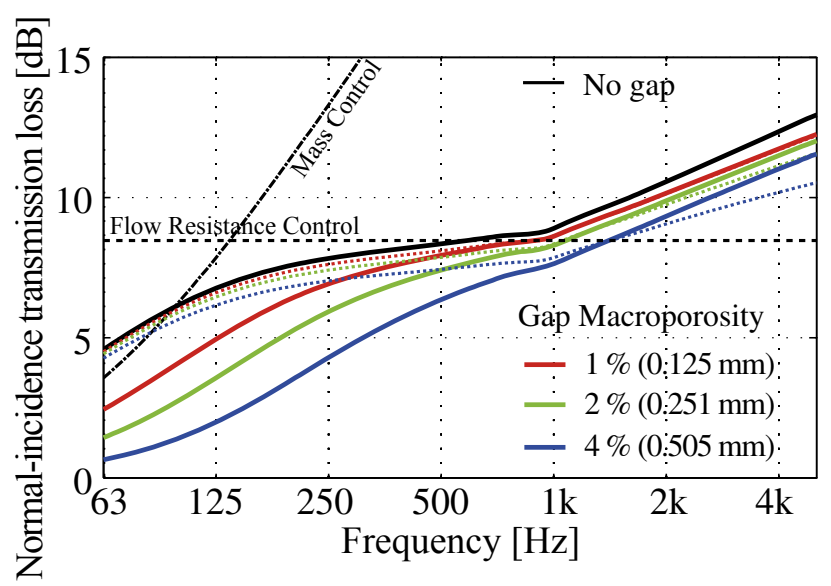

Fig. 8 Normal-incidence transmission losses calculated for the material with different circumferential gaps. Dotted lines represent the composite transmission losses weighted with 1,2 , and $4 \%$ macroporosity, where the transmission coefficients of the air gaps are assumed to be unity.

depends on the geometrical relations among the wavelength in the double porosity medium and the positions of the material and the hard backing.

\subsection{Transmission Loss}

Figure 8 shows transmission losses calculated for materials with different circumferential gaps. The material thickness is $25 \mathrm{~mm}$. Under the circumferential gapped condition, transmission losses decrease from those for the infinite-area material in the entire frequency range. As the gaps become larger, the tendency becomes more pronounced in the low-frequency range. By comparing the solid and dotted lines, it can be inferred that the circumferential gap gives the diffraction path and that the acoustic wave propagates to the transmission side mainly through the gap in the low-frequency range. The composite transmission losses ignore the attenuation in the gap. Hence, underestimation occurs at high frequencies.

\section{CONCLUSION}

The primary objective of the impedance tube method is to directly measure acoustical characteristics that are equivalent to those for the infinite-area material. The numerical investigation conducted here has confirmed that the measurement can be hindered by the effects of circumferential constraint and gapped conditions regarding the above objective. Under the circumferential constraint condition, the following tendencies were demonstrated for both absorption and transmission characteristics:

- Characteristics in the high-frequency range are hardly affected.

- The frame resonances occur around the shear resonance frequency except for the 12.5 -mm-thick mate-
Shear Modulus : $N$

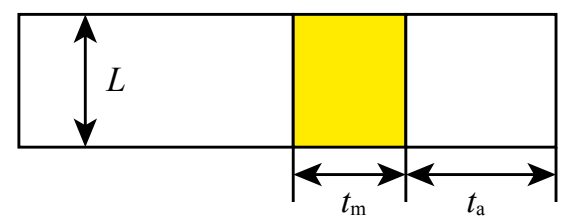

- Lateral faces of the material are fixed on the side wall of the tube.

- Making the air layer and the specimen as thick as possible is preferable.

- The shear modulus may be estimated from the peak frequency of the absorption coefficient as $N=2 L^{2} f_{\mathrm{s}}^{2} \rho_{\mathrm{b}}$.

Young's Modulus : $E$

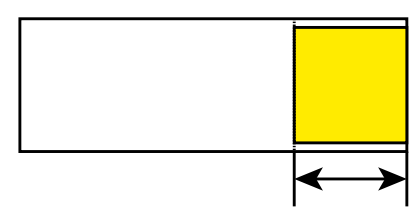

$t_{\mathrm{m}}$

- The material must be fixed on the bottom of the tube.

- Measurement is conducted under the circumferential gap condition.

- Young's modulus may be estimated from the dip frequency of the absorption coefficient as $E=16 t_{\mathrm{m}}^{2} f_{1}^{2} \rho_{\mathrm{b}}$.

Flow Resistivity : $\sigma$

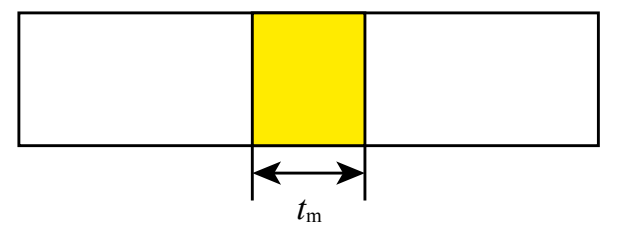

- The material is fixed on the side wall of the tube.

- The flow resistivity may be estimated from the low-frequency limit of the transmission loss by the following relation.

$$
R_{\mathrm{n}}=20 \log _{10}\left|1+\frac{\sigma t_{\mathrm{m}}}{2 \rho_{0} c_{0}}\right| \quad(\omega \rightarrow 0)
$$

Fig. 9 Summary of the measurement arrangements for the estimation of the mechanical and acoustical parameters of a poroelastic material.

rial. As the material becomes thinner, the frame resonance tends to occur around the bending resonance frequency for a thick plate.

- Characteristics below the resonance frequency of the frame approach those for the rigid frame. When there is an air layer behind the poroelastic material, the mass-spring resonance in the low-frequency range is restrained, which leads to an increase in the degree of discrepancy between the circumferential slip and constraint conditions. 
On the other hand, under the circumferential gapped condition, the following tendencies were demonstrated:

- In comparison with the ungapped condition, absorption coefficients decrease below and increase around the frequency at which the quarter wavelength in the double-porosity material becomes equal to the material thickness.

- These discrepancies become pronounced for the material with a back air layer.

- Transmission losses decrease in the entire frequency range and markedly decrease in the low-frequency range.

The actual size of the circumferential gap and the actual strength of circumferential constraint are unknown because it is practically difficult to control the size of the material precisely. As a result, the circumferential constraint condition may be preferable because the characteristics under this condition approach those for the rigidframe material except in the frequency range around the frame resonance. However, it should be kept in mind that the condition might involve compression of a specimen when it is set in a tube, which causes another uncertainty in ITM.

Moreover, there is a possibility that the mechanical and acoustical parameters of poroelastic materials can be estimated from the results measured under the circumferential constraint/gapped conditions. A summary of the estimation procedures is given in Fig. 9. Further work should be carried out to assess the validity and viability of these procedures in actual measurement.

\section{REFERENCES}

[1] ISO 10534-2 Acoustics - Determination of sound absorption coefficient and impedance in impedance tubes-Part 2: Transfer-function method (1998).

[2] Y. J. Kang and J. S. Bolton, "Finite element modeling of isotropic elastic porous materials coupled with acoustical finite elements," J. Acoust. Soc. Am., 98, 635-643 (1995).

[3] T. E. Vigran, L. Kelders, W. Lauriks, P. Leclaire and T. F. Johansen, "Prediction and measurement of the influence of boundary conditions in a standing wave tube," Acta Acust., 83, 419-423 (1997).

[4] B. H. Song, J. S. Bolton and Y. J. Kang, "Effect of circumferential edge constraint on the acoustical properties of glass fiber materials," J. Acoust. Soc. Am., 110, 2902-2916 (2001).

[5] D. Pilon, R. Panneton and F. Sgard, "Behavioral criterion quantifying the edge-constrained effects on foams in the standing wave tube," J. Acoust. Soc. Am., 114, 1980-1987 (2003).

[6] A. Cummings, "Impedance tube measurements on porous media: The effects of air-gaps around the sample," J. Sound Vib., 151, 63-75 (1991).

[7] D. Pilon, R. Panneton and F. Sgard, "Behavioral criterion quantifying the effects of circumferential air gaps on porous materials in the standing wave tube," J. Acoust. Soc. Am., 116, 344-356 (2004).

[8] X. Olny and C. Boutin, "Acoustic wave propagation in double porosity media," J. Acoust. Soc. Am., 114, 73-89 (2003).

[9] J. F. Allard and N. Atalla, Propagation of Sound in Porous Media: Modelling Sound Absorbing Materials, 2nd ed. (John Wiley \& Sons, 2009), Chap. 11.

[10] N. Atalla, R. Panneton and P. Debergue, "A mixed displacement-pressure formulation for poroelastic materials," $J$. Acoust. Soc. Am., 104, 1444-1452 (1998).

[11] Y. Champoux and J. F. Allard, "Dynamic tortuosity and bulk modulus in air-saturated porous media," J. Appl. Phys., 70, 1975-1979 (1991).

[12] A. Craggs, "A finite element model for rigid porous absorbing materials," J. Sound Vib., 61, 101-111 (1978).

[13] P. Debergue, R. Panneton and N. Atalla, "Boundary conditions for the weak formulation of the mixed $(u, p)$ poroelasticity problem," J. Acoust. Soc. Am., 106, 2383-2390 (1999).

[14] N. Inoue and T. Sakuma, "Numerical investigation on the support condition of the material in impedance tube measurement - Effect of the cross-sectional shapes of the tube," Proc. Annu. Meet., Environ. Eng. (Archit. Inst. Jpn.), pp. 155-156 (2012).

[15] N. Inoue, "Study on prediction of the performances of multilayered acoustic elements based on the vibro-acoustic numerical analysis," Ph.D. thesis, The University of Tokyo (2015) (in Japanese). 